\title{
Troponins in the definition of myocardial infarction after percutaneous coronary interventions
}

\author{
Marco Zimarino, Raffaele De Caterina \\ Institute of Cardiology and Center of Excellence on Aging, "G. d'Annunzio" University - Chieti, Italy
}

\section{ARTICLE INFO}

\section{Article history:}

Received: 23 September 2012

Accepted: 7 October 2012

Available online: 15 October 2012

Keywords:

Long-term outcome

Percutaneous coronary intervention

Periprocedural myocardial infarction

\begin{abstract}
The recently updated Universal Definition of Myocardial infarction (MI) arbitrarily defined periprocedural MI (type 4a) by elevation of cardiac troponin (cTn) values $>5 x$ the upper reference limit (URL) in patients with normal baseline values or a rise of cTn values $>20 \%$ if the baseline values are elevated, together with either angina or new ECG changes or angiographic loss of patency of a coronary artery or a side branch or persistent slow or no-flow or embolization, or imaging demonstration of new loss of viable myocardium. Although an association between CK-MB elevations and adverse prognosis after $\mathrm{PCl}$ has been documented, existing data do not however support the statement that an isolated elevation of $\mathrm{CTn}$ after $\mathrm{PCl}$ is associated with an adverse prognosis after $\mathrm{PCl}$; increased $\mathrm{CTn}$ levels before $\mathrm{PCl}$ seem far more predictive of future events than a periprocedural increase itself.

Caution should be paid in the interpretation of clinical trials using type $4 \mathrm{a} \mathrm{MI}$ as a primary endpoint. Nevertheless, patients with periprocedural myocardial damage should be treated as a higher-risk cohort, carefully monitored and receive an intensified secondary prevention program.
\end{abstract}

○ 2012, ČKS. Published by Elsevier Urban and Partner Sp. z o.o. All rights reserved.
Percutaneous coronary interventions ( $\mathrm{PCl})$, although successful in restoring vessel patency and relieving ischemia without angiographic complications, may provoke myocardial injury [1]. Periprocedural myocardial infarction (MI) is mostly caused by coronary dissection or side-branch occlusion at the site of intervention, or by distal microembolization of atherothrombotic debris downstream of the treatment site [2]. In the era of balloon angioplasty, embolization was considered a rare event, mainly confined to the treatment of lesions with extensive thrombus burden. Later on, with the development of the creatine kinase (CK) MB isoform and cardiac troponin (cTn) I or T assays - and the extensive use of coronary stents, neologisms such as "necrosettes", "enzyme leaks" and "infarctlets" [3] were coined, describing a seemingly uneventful finding.
With the aim of more precisely and comprehensively defining MI, a joint task force of the European Society of Cardiology (ESC), the American College of Cardiology Foundation (ACCF), the American Heart Association (AHA) and the World Heart Federation (WHF) has recently published an update of the "Universal Definition of Myocardial Infarction" [4]. MI occurring after PCl was defined as type $4 a$. In the 2007 version, the diagnosis of periprocedural Ml was suggested after an increase of any myocardial necrosis markers - either CTn or CK-MB - > 3 times the upper reference limit (URL) [5]. The recently updated 2012 version has dismissed CK-MB in favor of cTn, has taken into account a pre-procedural evaluation of $\mathrm{CTn}$, and coupled the need of biomarker increase with a clinico-strumental detection of myocardial "injury" to define MI in this setting. Periprocedural MI has been therefore arbi-

Address: Marco Zimarino, MD, PhD, Institute of Cardiology, "G. d'Annunzio" University - Chieti, C/o Ospedale SS. Annunziata, Via dei Vestini, 66100 Chieti, Italy, e-mail: m.zimarino@unich.it DOI: 10.1016/j.crvasa.2012.10.001 
Table 1 - Comparison of 2007 and 2012 versions of the universal definition of myocardial infarction after PCl - type 4a (as modified by Thygesen et al. [4-5]).

\section{7 version}

Increases $>3$ URL of either CK-MB or cTn.

If cardiac troponin is elevated before the procedure and not stable for at least 2 samples $6 \mathrm{~h}$ apart, there are insufficient data to recommend biomarker criteria for the diagnosis of peri-procedural MI.

\section{2 version}

In patients with normal cTn baseline value

- elevation of cTn values $>5$ times URL

If the baseline values are elevated

- rise of $c T n$ values $>20 \%$ together with

- angina or new ECG changes or

- angiographic loss of patency of a coronary artery or

a side branch or

- persistent slow or no-flow or embolization, or

- imaging demonstration of new loss of viable myocardium.

CTn - cardiac troponins; ECG - electrocardiogram; MI - myocardial infarction; PCI - percutaneous coronary interventions;

URL - upper reference limit.

trarily defined (Table 1) by elevation of cTn $>5$ times URL in patients with normal baseline values or a rise of cTn values $>20 \%$ if the baseline values are elevated, together with either angina or new ECG changes or angiographic loss of patency of a coronary artery or a side branch or persistent slow or no-flow or embolization, or imaging demonstration of new loss of viable myocardium.

After myocardial revascularization, signs of myocardial injury are extremely frequent: ST-segment and T wave abnormalities occur in about $20 \%$ of patients, but no prognostic relevance has ever here been documented [6]. On the contrary, an increase of CK-MB after PCl clearly affects late outcome $[7,8]$, but the currently widespread attitude to substitute CK-MB with CTn [9] together with a non-critical transfer of thresholds agreed upon for one to another - far more sensitive - biomarker is likely to cause an overdiagnosis of $\mathrm{MI}$ after $\mathrm{PCl}$ [10]. Moreover, the traditional interpretation of cTn appearance in the blood as being diagnostic of myocite membrane damage and therefore of myocardial necrosis has been recently challenged, as increasing levels of CTnT have been detected following a pacing-induced stress [11], or high-endurance exercise, without any evidence of scar by cardiac magnetic resonance imaging [12].

Conflicting evidence emerges in attempts at documenting that a periprocedural increase of cTn implies an independent increased risk of "solid" adverse outcomes. In a detailed review of the existing literature on this issue [13], we recently identified a strong association between $\mathrm{CTn}$ as a continuous value and hard events, but failed to document any association between increased CTn and long-term mortality or MI when levels of CK-MB after $\mathrm{PCl}$ are at the same time within the URL. Moreover, currently available high-sensitivity cTn assays are $10^{3}$ to $10^{4}$-fold more sensitive than the original first generation assays - on which most of the literature has been built and the isolated use of these newer assays may reduce the specificity of MI diagnosis [14]. In a limited series of patients undergoing cardiac magnetic resonance early after $\mathrm{PCl}$, procedure-related $\mathrm{MI}$ was detected by late gadolinium enhancement only when subjects experienced an increase of cTn $>40$ times URL [15]. Similarly, in a registry of 4,930 patients undergoing elective $\mathrm{PCl}$, a regression spline model documented that the hazard of 1-year mortality significantly increased for CK-MB rise $>3$ times URL, but only for CTn elevation > 20 times URL after PCI [16].

The inference of an adverse outcome for the sole increase of cTn after $\mathrm{PCl}$ seems to us not prudent: it is unlikely that such types of minor cardiac injury might affect long-term survival through a reduction in systolic function, which has never been documented in such cases. More plausible mechanisms might reside in electrical instability and repolarization abnormalities induced by myocellular damage, possibly provoking fatal arrhythmic events. We studied the long term implication of the changes in QT dispersion (QTD) - the interlead difference in QT commonly used as a measure of repolarisation inhomogeneity - in a consecutive series of patients undergoing elective $\mathrm{PCl}$ [17]. Successful PCI "normalizes" repolarization inhomogeneity, as reflected by a significant decrease of QTD after $\mathrm{PCl}$; on the other hand, a defective recovery of QTD is an independent predictor of long-term cardiac mortality. The combined detection of increased repolarization inhomogeneity and periprocedural MI might be extremely useful to risk-stratify the $\mathrm{PCl}$ population: in our series, patients with an increase in QTD $>2 \mathrm{~ms}$ and CK-MB $>$ URL after PCl experienced a 4-year cardiac mortality 6 times higher than the remaining population [17].

In conclusion, isolated increase of CTn after PCl over the currently proposed threshold of 5 times the URL - in the absence of any increase in CK-MB - is likely oversensitive, and cannot be considered an independent predictor of late adverse outcome, even in the presence of clinico-diagnostic signs of myocardial injury. The question whether we should call these biomarker increases "Mls" despite the lack of of a demonstration of their prognostic significance remains still unanswered at this time. Nonetheless, cTn levels should be routinely measured before and after PCl. Subjects with a preprocedural increase of cTn should be managed according to ACS guidelines; patients who experience a postprocedural rise of cTn should be closely monitored, and not considered for early discharge 
programs. However, as also suggested by Prasad and Herrmann [1], repeat coronary angiography should be recommended in patients with recurrent chest pain, particularly in combination with ST-segment shifts or echocardiographic evidence of ischemia or pericardial effusion.

\section{References}

[1] A. Prasad, J. Herrmann, Myocardial infarction due to percutaneous coronary intervention, The New England Journa of Medicine 364 (2011) 453-464.

[2] I. Porto, J.B. Selvanayagam, W.J. Van Gaal, et al., Plaque volume and occurrence and location of periprocedural myocardial necrosis after percutaneous coronary intervention: insights from delayed-enhancement magnetic resonance imaging, thrombolysis in myocardial infarction myocardial perfusion grade analysis, and intravascular ultrasound, Circulation 114 (2006) 662-669.

[3] D.R. Holmes, Jr., P.B. Berger, Troponisms, necrosettes, enzyme leaks, creatinine phosphokinase bumps, and infarctlets: what's behind this new lexicon and what does it add?, Circulation 104 (2001) 627-629.

[4] K. Thygesen, J.S. Alpert, A.S. Jaffe, et al., Third universal definition of myocardial infarction, European Heart Journal 33 (2012) 2551-2567.

[5] K. Thygesen, J.S. Alpert, H.D. White, Universal definition of myocardial infarction, European Heart Journal 28 (2007) 2525-2538.

[6] Y. Yokoyama, B.R. Chaitman, R.M. Hardison, et al., Association between new electrocardiographic abnormalities after coronary revascularization and five-year cardiac mortality in BARI randomized and registry patients, American Journal of Cardiology 86 (2000) 819-824.

[7] A.E. Abdelmeguid, E.J. Topol, P.L. Whitlow, et al., Significance of mild transient release of creatine kinase-MB fraction after percutaneous coronary interventions, Circulation 94 (1996) 1528-1536.

[8] C. Cavallini, S. Savonitto, R. Violini, et al., Impact of the elevation of biochemical markers of myocardial damage on long-term mortality after percutaneous coronary intervention: results of the CK-MB and PCl study, European Heart Journal 26 (2005) 1494-1498.

[9] A.K. Saenger, A.S. Jaffe, Requiem for a heavyweight: the demise of creatine kinase-MB, Circulation 118 (2008) 22002206.

[10] K.M. Eggers, L. Lind, P. Venge, B. Lindahl, Will the universal definition of myocardial infarction criteria result in an overdiagnosis of myocardial infarction?, American Journal of Cardiology 103 (2009) 588-591.

[11] A.T. Turer, T.A. Addo, J.L. Martin, et al., Myocardial ischemia induced by rapid atrial pacing causes troponin $T$ release detectable by a highly sensitive assay: insights from a coronary sinus sampling study, Journal of the American College of Cardiology 57 (2011) 2398-2405.

[12] N. Mousavi, A. Czarnecki, K. Kumar, et al., Relation of biomarkers and cardiac magnetic resonance imaging after marathon running, American Journal of Cardiology 103 (2009) 1467-1472.

[13] M. Zimarino, V. Cicchitti, E. Genovesi, et al., Isolated troponin increase after percutaneous coronary interventions: does it have prognostic relevance?, Atherosclerosis 221 (2012) 297-302.

[14] J.O. Baker, J. Reinhold, S. Redwood, M.S. Marber, Troponins: redefining their limits, Heart 97 (2011) 447-452.

[15] C.C. Lim, W.J. van Gaal, L. Testa, et al., With the "universal definition", measurement of creatine kinase-myocardial band rather than troponin allows more accurate diagnosis of periprocedural necrosis and infarction after coronary intervention, Journal of the American College of Cardiology 57 (2011) 653-661.

[16] V. Novack, M. Pencina, D.J. Cohen, et al., Troponin criteria for myocardial infarction after percutaneous coronary intervention, Archives of Internal Medicine 172 (2012) 502-508.

[17] M. Zimarino, A. Corazzini, A. Tatasciore, et al., Defective recovery of QT dispersion predicts late cardiac mortality after percutaneous coronary intervention, Heart 97 (2011) 466-472. 